\title{
New Exact Results and Bounds for Bipartite Crossing Numbers of Meshes ${ }^{\star}$
}

\author{
Matthew C. Newton ${ }^{1}$, Ondrej Sýkora ${ }^{1}$, Martin Užovič ${ }^{2}$, and Imrich Vrt'o ${ }^{3}$ \\ 1 Department of Computer Science, Loughborough University, \\ Loughborough, Leicestershire LE11 3TU, UK \\ $\{$ m.c.newton, o.sykora\}@lboro.ac.uk \\ 2 Department of Computer Science, Comenius University, \\ Mlynská dolina, 84248 Bratislava, Slovak Republic \\ 3 Department of Informatics, Institute of Mathematics, Slovak Academy of Sciences, \\ Dúbravská 9, 84104 Bratislava, Slovak Republic \\ vrto@savba.sk
}

\begin{abstract}
The bipartite crossing number of a bipartite graph is the minimum number of crossings of edges when the partitions are placed on two parallel lines and edges are drawn as straight line segments between the lines. We prove exact results, asymtotics and new upper bounds for the bipartite crossing numbers of 2-dimensional mesh graphs. We especially show that $\operatorname{bcr}\left(P_{6} \times P_{n}\right)=35 n-47$, for $n \geq 7$.
\end{abstract}

\section{Introduction}

The planar crossing number is the minimum number of edge crossings in any drawing of a graph in the plane. This is an important and difficult problem which has been studied in graph theory, as well as in the theory of VLSI [4, 10,15]. Computing the value of the planar crossing number is $N P$-hard [6], and exact values are known only for very restricted classes of graphs. In this paper we study a frequent variant of the planar crossing number. Let $G=\left(V_{0}, V_{1}, E\right)$ be a bipartite graph, where $V_{0}, V_{1}$ is the bipartition of vertices into independent sets. A bipartite drawing of $G$, denoted by $\mathcal{D}(G)$ consists of placing the vertices of $V_{0}$ and $V_{1}$ into distinct points on two horizontal lines $y=0, y=1$ in the $x y$-plane, respectively, and then drawing each edge with one straight line segment which connects the end-vertices. The bipartite crossing number of the drawing, denoted by $\operatorname{bcr}(\mathcal{D}(G))$, is the number of crossing pairs of edges in the drawing. The bipartite crossing number of $G$, denoted by $\operatorname{bcr}(G)$, is the minimum $\operatorname{bcr}(\mathcal{D}(G))$ over all drawings.

A motivation behind studying $\operatorname{bcr}(G)$ comes from the routing of VLSI (see for example $[10,17])$. Another motivation appears in the field of graph drawing. It is well known that $\operatorname{bcr}(G)$ is one of the parameters which strongly influences the understanding and aesthetics of drawings of graph-like structures, especially in a hierarchical fashion. For a survey on drawing graphs see [3].

* This research was supported by the EPSRC grant GR/R37395/01 and by VEGA grant No. 2/3164/23. 
The notion of $\operatorname{bcr}(G)$ was first introduced in [7], [8] and [23] where it was stated that $\operatorname{bcr}(G)=0$ iff the graph is caterpillar, and $\operatorname{bcr}\left(C_{n}\right)=n / 2-1$ for an $n$-vertex cycle ( $n$ is even). Some basic observations on $\operatorname{bcr}(G)$ were made in [13]. The bipartite crossing number problem is known to be NP-complete [6] but can be solved in polynomial time for bipartite permutation graphs [20], and trees [18]. A great deal of research has been devoted to the design of algorithms and heuristics for solving this problem (see for example $[2,5,9,11,12,14,21,22]$ ).

To find exact results of $\operatorname{bcr}(G)$ for special families of graphs is of interest from the graph-theoretic point of view, but such results can also be applied in testing heuristics for bipartite drawings. So far the heuristics are mostly compared against each other but not against the optimal drawing, which is typicaly unknown. For this purpose one can use

$$
\operatorname{bcr}\left(S\left(K_{n}\right)\right)=4\left(\begin{array}{l}
n \\
4
\end{array}\right)+2\left(\begin{array}{l}
n \\
3
\end{array}\right),
$$

where $S\left(K_{n}\right)$ denotes the complete graph with exactly one new vertex on every edge [8]. They proved a similar formula for the subdivided complete bipartite graph. In [18] we proved for the complete binary tree $T_{n}$ of depth $n-1$

$$
\operatorname{bcr}\left(T_{n}\right)=\frac{1}{9}\left((3 n-11) 2^{n}+2(-1)^{n}\right)+2 .
$$

For the mesh $P_{m} \times P_{n}$, i.e. the graph defined by the Cartesian product of an $m$-vertex path with an $n$-vertex path, where $3 \leq m \leq n$, we found in [19] $\operatorname{bcr}\left(P_{3} \times P_{n}\right)=5 n-6$, for $n \geq 3$. To our knowledge these are all known nontrivial exact results for the bipartite crossing number of typical graphs. In [19] we also proved an estimation

$$
\frac{3}{4} m(m-18) n+O\left(m^{3}\right) \leq \operatorname{bcr}\left(P_{m} \times P_{n}\right) \leq \frac{3}{2} m(m-1) n .
$$

In this paper we prove an asymptotic

$$
\lim _{n \rightarrow \infty} \frac{\operatorname{bcr}\left(P_{m} \times P_{n}\right)}{n}=\frac{1}{2}\left(3 m^{2}-7 m+4\right)
$$

and exact results for $m=4,5$, and 6. Especially,

$$
\operatorname{bcr}\left(P_{6} \times P_{n}\right)=\left\{\begin{array}{l}
161, \text { if } n=6, \\
35 n-47, \text { if } n \geq 7 .
\end{array}\right.
$$

We conclude the paper with new upper bounds for bipartite crossing numbers of general meshes and some conjectures about exact values.

\section{A General Lower Bound}

In this section we prove a lower bound for general meshes. Assume that the mesh has $m$ horizontal rows and $n$ vertical columns. 
Theorem 1. For a mesh $P_{m} \times P_{n}, n>8 m$ :

$$
\operatorname{bcr}\left(P_{m} \times P_{n}\right) \geq \frac{1}{2}\left(3 m^{2}-7 m+4\right) n-4 m^{3}+\frac{21}{2} m^{2}-\frac{7}{2} m .
$$

Proof. Let us have a bipartite drawing $\mathcal{D}$ of the mesh. Divide the drawing into three parts: $\mathcal{D}_{1}, \mathcal{D}_{2}, \mathcal{D}_{3}$. Define $\mathcal{D}_{1}$ as a part of drawing constructed in the following way: starting from the left end of the drawing, take alternate vertices from both partitions until this set of vertices contains vertices from $m$ different columns of the mesh. The convex hull of this set is $\mathcal{D}_{1}$. Starting from the right end of the drawing we similarly construct the part $\mathcal{D}_{3}$ until it contains vertices of $m$ different columns (different also from those in the $\mathcal{D}_{1}$ ). The rest is $\mathcal{D}_{2}$. From the Dirichlet principle it follows that the total number of vertices in $\mathcal{D}_{1}$ and $\mathcal{D}_{3}$ is at most $2 m^{2}$, and the number of horizontal edges incident to the vertices from $\mathcal{D}_{1}$ and $\mathcal{D}_{3}$ is at most $4 m^{2}$. Now, choose $m$ vertices of different columns from $\mathcal{D}_{1}$ and $m$ vertices of different columns in $\mathcal{D}_{3}$. Take a bijection between these two groups of vertices and for any pair join its vertices by a path that uses exclusively edges of one row of the mesh and the edges in the columns where the vertices of the pair reside. In Fig. 1 there is such a path, shown by the heavy line. The path can use edges of the mesh as shown in Fig. 2 . The $m$ paths are obviously vertex disjoint.

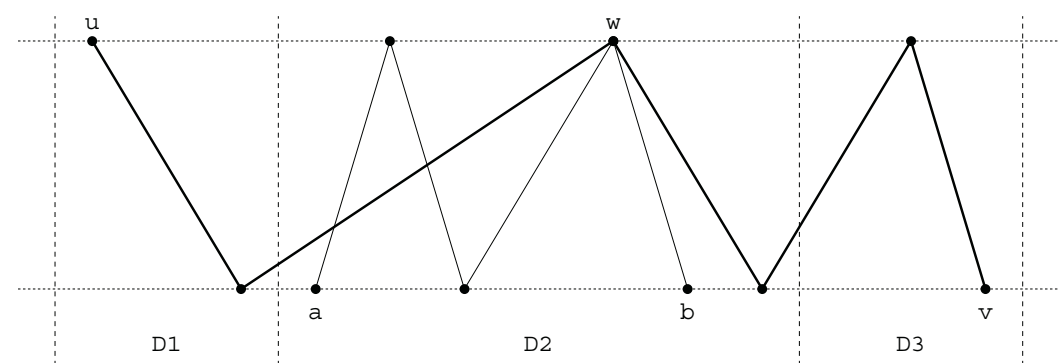

Fig. 1. An example of a path between vertices $u$ and $v$, which crosses the column $a-b$ in vertex $w$.

We have $m$ such edge disjoint paths. There are at least $n-2 m$ complete columns in $\mathcal{D}_{2}$. Now we count the number of crossings of the above $m$ paths with edges of these columns. There are $m-1$ column edges in one column and at least $m-2$ paths must cross each of them (two paths may use end-vertices of a column edge). Therefore there are $(n-2 m)(m-1)(m-2)$ crossings of this type.

If a row edge lies completely in $\mathcal{D}_{2}$ it must be crossed by $m-1$ paths as one path can use the edge. As there are at least $(n-1) m-4 m^{2}$ such edges, we have at least $\left((n-1) m-4 m^{2}\right) \times(m-1) / 2$ crossings of this type $(2$ in denominator is because a crossing is counted twice), which in total gives the claimed lower bound.

In Section 4 we describe an upper bound which coincides with this lower bound up to the second order term for fixed $m$ and $n>>m$ which implies: 


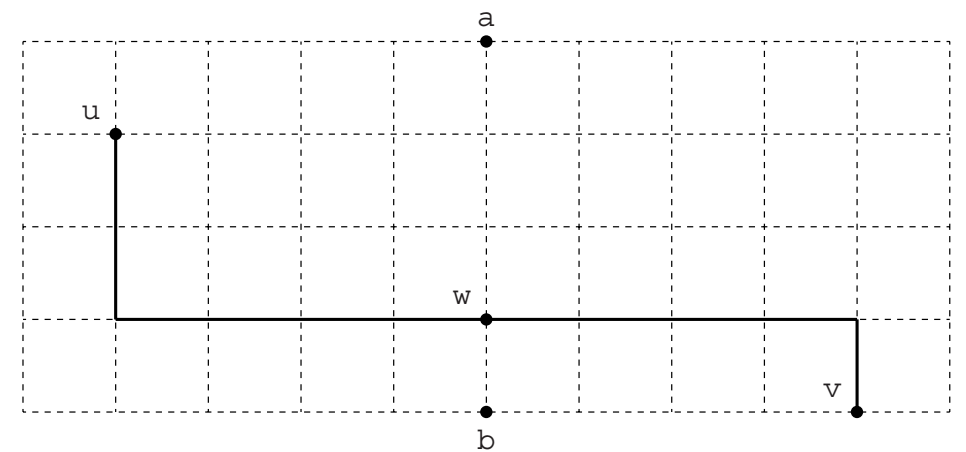

Fig. 2. Example of the path between the vertices $u$ and $v$ in the mesh.

Theorem 2. For any fixed $m \geq 3$

$$
\lim _{n \rightarrow \infty} \frac{\operatorname{bcr}\left(P_{m} \times P_{n}\right)}{n}=\frac{1}{2}\left(3 m^{2}-7 m+4\right)
$$

\section{Three Exact Results}

Here we prove exact results on the bipartite crossing numbers for $m=4,5,6$. We will skip the (simpler) proofs for the cases $m=4,5$.

Theorem 3. The bipartite crossing numbers of $P_{4} \times P_{n}, P_{5} \times P_{n}$ and $P_{6} \times P_{n}$ satisfy

$$
\begin{aligned}
& \operatorname{bcr}\left(P_{4} \times P_{n}\right)=\left\{\begin{array}{l}
33, \text { if } n=4, \\
12 n-14, \text { if } n \geq 5 .
\end{array}\right. \\
& \operatorname{bcr}\left(P_{5} \times P_{n}\right)=\left\{\begin{array}{l}
81, \text { if } n=5, \\
22 n-28, \text { if } n \geq 6 .
\end{array}\right. \\
& \operatorname{bcr}\left(P_{6} \times P_{n}\right)=\left\{\begin{array}{l}
161, \text { if } n=6, \\
35 n-47, \text { if } n \geq 7 .
\end{array}\right.
\end{aligned}
$$

Proof. The matching upper bounds will be shown in Section 4. It is known that $\operatorname{bcr}\left(P_{6} \times P_{6}\right)=161$ and $\operatorname{bcr}\left(P_{6} \times P_{7}\right)=198$. The values were found by Thomas Odenthal using a branch and bound algorithm [16].

The leftmost 'comb', $C$, is a sub-graph of the mesh defined as a graph induced by the first two columns without the edges of the second column. Similarly we define the rightmost comb. Let us call the convex hull of the drawing of $C$ the comb region, $R$.

We proceed by induction on $n$. Let the claim hold for $n-1 \geq 7$. Consider an optimal drawing $\mathcal{D}\left(P_{6} \times P_{n}\right)$. If there are 35 crossings on the leftmost or rightmost comb then by deleting that comb we get a drawing $\mathcal{D}\left(P_{6} \times P_{n-1}\right)$. Hence

$$
\operatorname{bcr}\left(\mathcal{D}\left(P_{6} \times P_{n}\right)\right) \geq 35+\operatorname{bcr}\left(\mathcal{D}\left(P_{6} \times P_{n-1}\right)\right) \geq 35 n-47 .
$$


However, if both combs contain less than 35 crossings each, our aim is to show that the drawing must be in special forms for which we determine the numbers of crossings directly. Roughly speaking, in this case both combs must be placed in "extreme" positions (left and right) in the drawing. Let $Z$ be the set of vertices which are not in the left comb $C$. Assume that there are at least two vertices, or one vertex of at least degree 3 , of $Z$ to the left of $R$, and at least two vertices, or one vertex of at least degree 3 , of $Z$ to the right of $R$. Then there exists at least 3 edge disjoint paths between these vertices in the mesh, which are vertex disjoint from $C$. As $C$ has 11 edges, the 3 paths force 33 crossings on $C$. Note that there must also be 5 additional crossings on $C$ caused just by the edges of $C$ with edges of the second column. In total we have 38 crossings on $C$, a contradiction.

WLOG we assume that there is at most one vertex $v \in Z$ of degree two lying to the left of $R$. Let $S \subseteq Z$ denote the set of vertices lying in the comb region $R$. We claim that there are at least 17 vertices of $Z$, which are from the 3rd to the 8th columns, lying to the right of the comb region $R$. If there are only at most 16 such vertices, then as $n \geq 8$, the graph induced by $Z$ has at least 60 edges and at most 24 of them are in the graph induced by those 16 vertices. Hence the number of edges incident to $S$ is at least 36 and every such edge produces a crossing on $C$, a contradiction.

Take the set $Z^{\prime}$ of 11 vertices out of those 17 such that they lie in the $3 \mathrm{rd}$ to the 7 th columns. One can show that there are 6 internally vertex disjoint paths, forming a set $\mathcal{P}$, between the vertices of the second column and vertices of $Z^{\prime}$, disjoint from the edges of $C$ and from the rightmost column. It implies that there is no vertex of degree 2 on the paths from $\mathcal{P}$.

If $S=\emptyset$, then we can move the degree two vertex $v$ from the left to the immediate right of $R$ without increasing crossings. We checked by exhaustive search on computer all possible drawings of $C$ (i.e. $(6 !)^{2}$ permutations) and found that if $S=\emptyset$ then the edges of $C$ are crossed at least 32 times. The crossings are caused either by paths from $\mathcal{P}$, or edges of the second column, or by $C$ edges with $C$ edges. Moreover, there is only one drawing of $C$ with precisely 32 crossings, shown in Fig. 3. We call it 'optimal with empty $S$ '.

The notation and type of lines we use for vertices and edges is shown in Fig. 4.

Assume that $S \neq \emptyset$ and that there are less than 35 crossings on the comb, $C$, i.e. its number of crossings is 33 or 34 .

Suppose there is a vertex of degree two in $S$. As it does not belong to any of the paths of $\mathcal{P}$, it causes at least 2 crossings more on $C$.

Suppose there is a vertex of degree three (or four) in $S$. It may belong to one of the paths of $\mathcal{P}$. This implies at least one (or two) more crossings on $C$. Consequently, if there are two nodes in $S$ and at least one of them is not of degree 3 , then there are at least 3 new crossings, i.e. at least 36 crossings on $C$, a contradiction.

Similarly, we get a contradiction if there is a single degree two vertex of $Z$ to the left of $R$, and there is a vertex in $S$. 


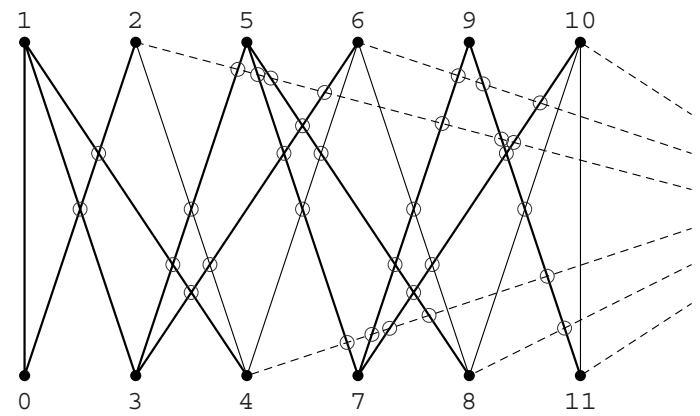

Fig. 3. Optimal drawing of the comb $C$ with empty $S$.

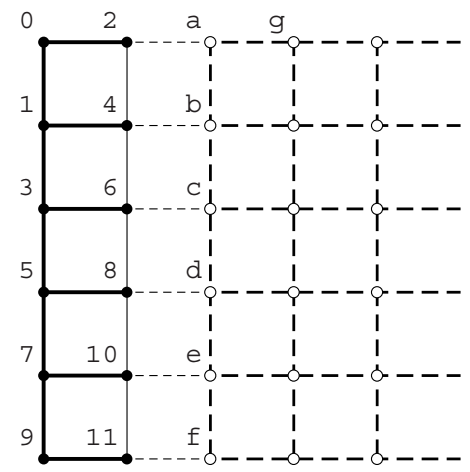

Fig. 4. Notation used for vertices of the comb $C$.

Let there be exactly one vertex of degree two or four in $S$. Such a vertex can be moved to the immediate right of the region $R$ without increasing the number of crossings.

Now, assume we have two vertices of degree 3 in $S$. The two vertices must be either both from the third column or next to each other from the same row (either first or sixth), otherwise we have at least 3 more crossings on $C$, a contradiction.

By a detailed case analysis one can show that in both situations the vertices can be moved to the immediate right of $R$ without increasing the number of crossings, i.e. all vertices of $Z$ will be to the right of $R$.

The last case we need to analyse is where there is a single degree 3 vertex in $S$. We checked by exhaustive search on computer all possible drawings of $C$ (i.e. $7(6 !)^{2}$ situations) and found that if there is one vertex of degree 3 in $S$ then $C$ is crossed at least 33 times, and there is only one such drawing of $C$ with precisely 33 crossings. We call it 'optimal with one vertex in $S$ ' (see Fig. 5).

So we conclude that the drawing of the left comb is either optimal with empty $S$ with 32 crossings or optimal with one vertex in $S$ with 33 crossings. We repeat the above arguments for the right comb.

Let us first have both combs in optimal drawings with empty $S$. In total they contain 64 crossings. Consider now 6 horizontal paths, forming a set $\mathcal{P}^{\prime}$, 


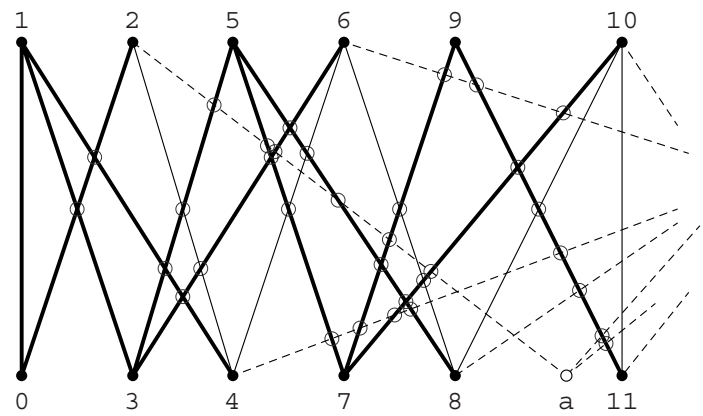

Fig. 5. Optimal drawing of the comb $C$ with one degree 3 vertex in $S$.

starting in the second column and ending in the $(n-1)$ th column. Similarly as in the proof of Theorem 1 , the paths from $\mathcal{P}^{\prime}$ create $20(n-4)$ crossings on the $3 \mathrm{rd}, \ldots,(n-2)$ nd column.

Now, we count the number of crossings caused mutually on paths from $\mathcal{P}^{\prime}$. Recall [8] that the bipartite crossing number of a $2 p$-vertex cycle is $p-1$. The paths of $\mathcal{P}^{\prime}$ are divided into 2 groups according to the line on which they start on the left side. Consider any 2 paths from different groups. Join them by 2 new artificial edges on the left and right side, respectively. We get a $(2 n-4)$-vertex cycle. As there are no crossings on the new edges the original paths cross at least $n-3$ times and the number of crossings between the paths of different groups is $9(n-3)$.

Consider any 2 paths from the same group. Identify the starting vertices of the paths on the left and right side, respectively. This operation does not increase the crossing number. We get a $(2 n-6)$-vertex cycle with $n-4$ crossings. Hence there are $3(n-4)$ crossings between the paths of each group. Altogether the number of mutal crossings on the paths is $15 n-51$.

The optimal drawing of the left comb $C$ forces 10 crossings between the edges of the 2 nd column and edges joining the 2 nd and the 3 rd column. A similar argument holds for the right hand side comb. So we have 20 such crossings. In total the number of crossings are

$$
\operatorname{bcr}\left(\mathcal{D}\left(P_{6} \times P_{n}\right)\right)=64+20(n-4)+15 n-51+20=35 n-47 .
$$

If, instead of being optimal with empty $S$ a comb is in the form optimal with one vertex in $S$, we have 33 crossings on the comb. We then have one less crossing:

- on edges of the third column with edges between the second and the third columns;

- on the edges between the second and the third columns with themselves; and

- on the edges of the second column with the edges between the second and the third columns.

We have one more crossing on: 
- the edges of the second and the third column; and

- on the the edges of the second column with the edges between the third and the fourth columns.

The changes in comparison with 'optimal drawing with empty $S$ ' are only local and they give the same number of crossings in total.

\section{Upper Bounds}

First we describe 2 types of bipartite drawings of meshes.

- A mesh $P_{m} \times P_{n}$ is drawn in a diagonal manner if the vertices are placed in the order shown in Fig. 6 .

We denote it by $\mathcal{D}_{d}\left(P_{m} \times P_{n}\right)$.

- We say that the mesh is drawn in a combined way if the vertices are placed in the order as shown in Fig. 7.

The parameter $s<n / 2$ denotes the number of left and right columns whose vertices are drawn in diagonal manner. We denote this type of drawing by $\mathcal{D}_{c}\left(P_{m} \times P_{n}\right)$.

Note that if $s=1$, the drawing is just a column by column drawing. One can check that the number of vertices in the above defined types of drawings of meshes is given by the following upper bounds:

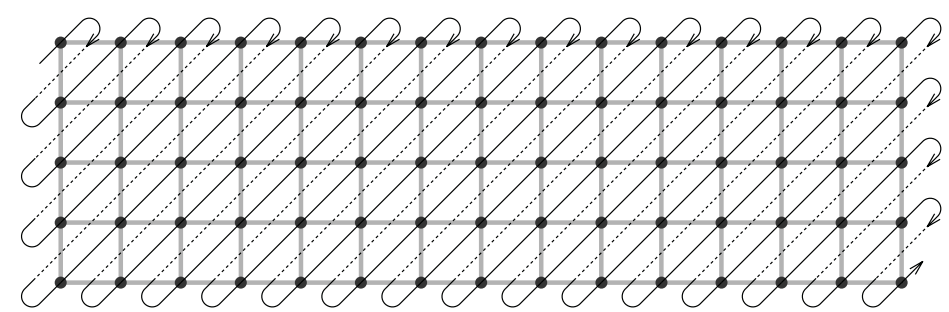

Fig. 6. Vertex order of mesh drawn in diagonal way.

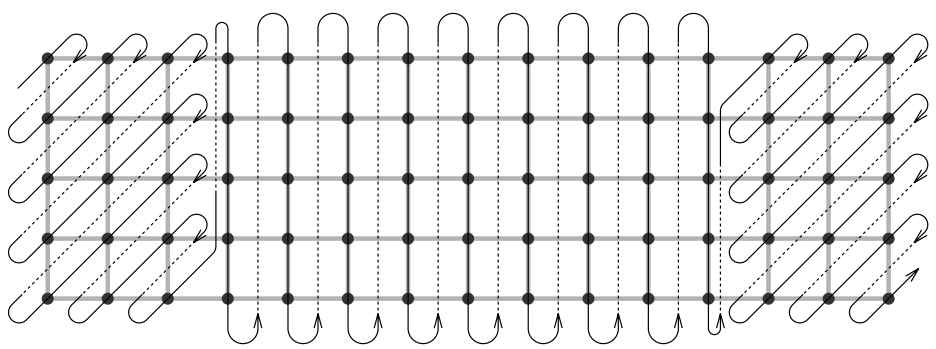

Fig. 7. Vertex order of mesh drawn in combined way. 
Theorem 4. Let $4 \leq m \leq n$. Then

$$
\begin{aligned}
& \operatorname{bcr}\left(\mathcal{D}_{d}\left(P_{m} \times P_{n}\right)\right)=\left(2 m^{2}-6 m+5\right) n-\frac{1}{3}\left(2 m^{3}-6 m^{2}+7 m-3\right), \\
& \operatorname{bcr}\left(\mathcal{D}_{c}\left(P_{m} \times P_{n}\right)\right)= \frac{1}{2}\left(3 m^{2}-7 m+4\right) n \\
&-\left\lceil\frac{(4 s+3) m^{2}}{4}\right\rceil+\left(2 s^{2}+s+1\right) m-\frac{1}{3} s(2 s-1)(s+5) .
\end{aligned}
$$

As a consequence we get matching upper bounds to our lower bounds from Section 3:

$$
\begin{aligned}
& \operatorname{bcr}\left(P_{4} \times P_{n}\right)=\left\{\begin{array}{l}
\operatorname{bcr}\left(\mathcal{D}_{d}\left(P_{4} \times P_{4}\right)\right)=33, \\
\operatorname{bcr}\left(\mathcal{D}_{c}\left(P_{4} \times P_{n}\right)\right)=12 n-14, \text { if } n \geq 5, \text { with } s=2 .
\end{array}\right. \\
& \operatorname{bcr}\left(P_{5} \times P_{n}\right)=\left\{\begin{array}{l}
\operatorname{bcr}\left(\mathcal{D}_{d}\left(P_{5} \times P_{5}\right)\right)=81, \\
\operatorname{bcr}\left(\mathcal{D}_{c}\left(P_{5} \times P_{n}\right)\right)=22 n-28, \text { if } n \geq 6, \text { with } s=2 .
\end{array}\right. \\
& \operatorname{bcr}\left(P_{6} \times P_{n}\right)=\left\{\begin{array}{l}
\operatorname{bcr}\left(\mathcal{D}_{d}\left(P_{6} \times P_{6}\right)\right)=161, \\
\operatorname{bcr}\left(\mathcal{D}_{c}\left(P_{6} \times P_{n}\right)\right)=35 n-47, \text { if } n \geq 7, \text { with } s=2 .
\end{array}\right.
\end{aligned}
$$

At this place we reproduce a table of exact results for small meshes obtained by Thomas Odenthal [16] using a branch and bound method.

\begin{tabular}{|c||c|c|c|c|c|}
\hline$m$ vs. $n$ & 4 & 5 & 6 & 7 & 8 \\
\hline \hline 4 & 33 & 46 & 58 & 70 & 82 \\
\hline 5 & & 81 & 104 & 126 & 148 \\
\hline 6 & & & 161 & 198 & 233 \\
\hline
\end{tabular}

The values for $m=n$ are obtained by diagonal drawings. The values for $n>m$ are obtained by combined drawings. In cases $m=5,6,7$ and $m=8$ and $n>m$ we use the combined drawing with $s=2$ and $s=3$, respectively.

For general square meshes the upper bound (1) gives

$$
\operatorname{bcr}\left(P_{m} \times P_{m}\right) \leq \frac{1}{3}\left(4 m^{3}-12 m^{2}+8 m+3\right),
$$

which we believe to be optimal.

For general rectangular meshes with $4 \leq m \leq n$, we have

$$
\operatorname{bcr}\left(P_{m} \times P_{m}\right) \leq \min \left\{\operatorname{bcr}\left(\mathcal{D}_{d}\left(P_{m} \times P_{n}\right)\right), \operatorname{bcr}\left(\mathcal{D}_{c}\left(P_{m} \times P_{n}\right)\right)\right\}
$$

The righthand side of the inequality (3) is minimized for a value $s=s_{m}$, for which as $m \rightarrow \infty, s_{m} / m \rightarrow 1-1 / \sqrt{2}=0.2928932 \cdots$. Comparing the minimum with the value for the diagonal drawing (1) we see that the diagonal drawing is better only in a narrow interval $m \leq n \leq \alpha_{m} m$, for which if $m \rightarrow \infty$, $\alpha_{m} \rightarrow 2(3-\sqrt{2}) / 3=1.057333 \cdots$. 


\section{Acknowledgment}

The authors thank Thomas Odenthal who found exact numbers for small size meshes.

\section{References}

1. Chung, F.R.K., A conjectured minimum valuation tree, SIAM Review 20 (1978), 601-604.

2. Demetrescu, C., Finocchi, I., Removing cycles for minimizing crossings, ACM Journal of Experimental Algorithms 6 (2001).

3. Di Battista, J., Eades, P., Tamassia, R., Tollis, I. G., Graph Drawing, Algorithms for the Visualization of Graphs, Prentice Hall, 1999.

4. Erdös, P., Guy, R. P., Crossing number problems, American Mathematical Monthly 80 (1973), 52-58.

5. Eades, P., Wormald, N., Edge crossings in drawings of bipartite graphs, Algorithmica 11 (1994), 379-403.

6. Garey, M. R., Johnson, D. S., Crossing number is NP-complete, SIAM Journal on Algebraic and Discrete Methods 4 (1983), 312-316.

7. Harary, F., Determinants, permanents and bipartite graphs, Mathematical Magazine 42 (1969), 146-148.

8. Harary, F., Schwenk, A., A new crossing number for bipartite graphs, Utilitas Mathematica 1 (1972), 203-209.

9. Jünger, M., Mutzel, P., 2-layer straight line crossing minimization: performance of exact and heuristic algorithms, Journal of Graph Algorithms and Applications 1 (1997), 1-25.

10. Leighton, F. T., Complexity Issues in VLSI, MIT Press, 1983.

11. Martí, R., A tabu search algorithm for the bipartite drawing problem, European Journal of Operational Research 106 (1998), 558-569.

12. Matuszewski, C., Schönfeld, R., Molitor, P., Using sifting for $k$-layer crossing minimization, in: Proc. 7th. Intl. Symposium on Graph Drawing, Lecture Notes in Computer Science 1731, Springer, Berlin, 2001, 217-224.

13. May, M., Szkatula, K., On the bipartite crossing number, Control and Cybernetics 17 (1988), 85-98.

14. Newton, M., Sýkora, O., Vrto, I., Two new heuristics for two-sided bipartite graph drawings, in: Proc. 10th Intl. Symposium on Graph Drawing, Lecture Notes in Computer Science 2528, Springer, Berlin, 2002, 312-319.

15. Pach, J., Agarwal, P. K., Combinatorial Geometry, Wiley and Sons, New York, 1995.

16. Odenthal, T., Personal communication, 2002.

17. Sarrafzadeh, M., An Introduction to VLSI Physical Design, McGraw-Hill, New York, 1995.

18. Shahrokhi, F., Sýkora, O., Székely, L. A., Vrto, I.: On the bipartite drawings and the linear arrangement problem, 5th Intl. Workshop on Algorithms and data Structures, Lecture Notes in computer Science 1272, Springer, Berlin, 1997, 55-68.

19. Shahrokhi, F., Sýkora, O., Székely, L. A., Vrto, I., A new lower bound for the bipartite crossing number with applications, Theoretical Computer Science $\mathbf{2 4 5}$ (2000), 281-294. 
20. Spinrad, J., Brandstädt, A., Stewart, L., Bipartite permutation graphs, Discrete Applied Mathematics 19 (1987), 279-292.

21. Sugiyama, K., Tagawa, S., Toda, M., Methods for visual understanding of hierarchical systems structures, IEEE Transactions on Systems, Man and Cybernetics 11 (1981), 109-125.

22. Warfield, J., Crossing theory and hierarchy mapping, IEEE Transactions on Systems, Man and Cybernetics 7 (1977), 502-523.

23. Watkins, M. E., A special crossing number for bipartite graphs: a research problem, Annals of New York Academy Sciences 175 (1970), 405-410. 